\title{
High Energy Gamma-Ray Emission from the Coma Cluster Region: Deep Morphological and Spectral Studies
}

\author{
Davit Zargaryan, ${ }^{a, b, *}$ Vardan Baghmanyan, ${ }^{c}$ Felix Aharonian, ${ }^{a, b, d}$ Jonathan \\ Mackey, ${ }^{a, b}$ Sabrina Casanova ${ }^{c, d}$ and Ruizhi Yang ${ }^{e}$ \\ ${ }^{a}$ Dublin Institute for Advanced Studies, \\ 31 Fitzwilliam Place, Dublin 2, Ireland \\ ${ }^{b}$ Centre for AstroParticle Physics and Astrophysics, DIAS Dunsink Observatory, \\ Dunsink Lane, Dublin 15, Ireland \\ ${ }^{c}$ Institute of Nuclear Physics PAN, \\ Radzikowskiego 152, 31-342 Kraków, Poland \\ ${ }^{d}$ Max-Planck-Institut für Kernphysik, \\ P.O. Box 103980, 69029 Heidelberg, Germany \\ ${ }^{e}$ Department of Astronomy, School of Physical Sciences, University of Science and Technology of China, \\ Hefei, Anhui 230026, China \\ E-mail: dzargaryan@cp.dias.ie, vardan.baghmanyan@ifj.edu.pl
}

The Coma galaxy cluster is one of the largest gravitationally-bound astrophysical structures in the local Universe, with a linear size of more than $2 \mathrm{Mpc}$ and a $\mathrm{z}=0.023$ distance. The relatively high intracluster density and the high-velocity accretion shocks within the cluster make it a possible source of high energy (HE) $\gamma$-ray emission. Using 12.3 years of Fermi-LAT Pass 8 data between $100 \mathrm{MeV}$ and $1 \mathrm{TeV}$ energies, we report the detection of HE diffuse $\gamma$-ray emission from the direction of the Coma cluster with $5.4 \sigma$ extension significance and $\mathrm{R}_{68}=0.82^{\circ}$ extension described with a homogeneous disk model. The corresponding $\gamma$-ray spectrum extends up to $\sim 50$ $\mathrm{GeV}$ with power-law spectral index $\Gamma=2.23 \pm 0.11$ and energy flux of $(3.48 \pm 0.68) \times 10^{-12}$ $\mathrm{erg} \mathrm{cm}^{-2} \mathrm{~s}^{-1}$. Furthermore, three point-like structures with power-law indexes of $2.44 \pm 0.28$, $2.56 \pm 0.32$, and $1.99 \pm 0.30$ show improvement in the fit by $\Delta_{\mathrm{AIC}}=7.2$ indicating more $\gamma$-ray sources in the region.

$37^{\text {th }}$ International Cosmic Ray Conference (ICRC 2021)

July 12 th - 23rd, 2021

Online - Berlin, Germany

\footnotetext{
${ }^{*}$ Presenter
} 


\section{Introduction}

Clusters of Galaxies are the largest astrophysical structures in the Universe where a variety of astronomical phenomena can be discussed [19]. One of the best examples is the Coma galaxy cluster (Abell 1656), which is considered a unique astronomical laboratory because of its proximity $(\mathrm{z}=0.023 ;[18])$, size (linear size of more than $2 \mathrm{Mpc})$ and mass $\left(M \sim 10{ }^{15} \mathrm{M}_{\odot}\right)$. It has been actively studied in all wavebands. For example, radio observations have shown radio halo and relic extended structures [10,12,14, 16, 20], which can be an indication of dense and high-energy accelerated particles accumulated in that region. Also, extended and rich morphological structures are visible in the X-ray band where relatively uniform temperature distribution and halo/relic structures are noticeable $[5,9,11,17]$.

In the high energy $\gamma$-ray band several studies have been made, starting in 2009 with HESS ( $>100 \mathrm{GeV}$ ) [4], where only flux upperlimits were estimated $F_{\gamma}<(0.1-6.1) \times 10^{-13} \mathrm{ph} \mathrm{cm}^{-2} \mathrm{~s}^{-1}$ in 1-10 TeV energy band. Next, analyzing six years of Fermi-LAT data, Ackermann et al. [2] found low significance residual $\gamma$-ray emission and an upper limit (UL) for the flux above $100 \mathrm{MeV}$, $F_{\gamma} \leq 1.7 \times 10^{-9} \mathrm{ph} \mathrm{cm}^{-2} \mathrm{~s}^{-1}$, was calculated. More recently, the $\gamma$-ray emission from Coma Cluster in the 0.2-300 GeV energy band have been detected Adam et al. [3], Xi et al. [22], where the $\gamma$-ray flux inside viral radius has estimated as $F_{\gamma} \sim 2 \times 10^{-12} \mathrm{erg} \mathrm{cm}^{-2} \mathrm{~s}^{-1}$ with a soft photon index of $\Gamma \sim 2.7$.

Motivated by broadband observations of this galaxy cluster and, in particular, the recent $\gamma$-ray detection with Fermi-LAT, we present a deep morphological and spectral investigation of $\gamma$-ray emission from the Coma region using more than 12 years of Fermi-LAT data.

\section{Fermi-LAT Gamma-Ray Data Analysis}

We analyzed Fermi-LAT Pass 8 data taken from August 4, 2008, to June 1, 2021, [6], in the energy range of between $100 \mathrm{MeV}$ and $1 \mathrm{TeV}$. We used ULTRACLEANVETO event class to study the low-level diffuse $\gamma$-ray emission from Coma Cluster. The data was taken within a region of interest (ROI) of $12^{\circ}$ around the centre of the Coma Cluster at R.A., Dec $=(194.95$, 27.98). We implemented $\gamma$-ray data analysis using Fermipy (version 1.0.0; Wood et al. 21) and Fermitools software packages v2.0.01. As recommended for the data analysis, we followed the standard binned-likelihood analysis technique for performing data reduction. For example, we used (DATA_QUAL $>0) \& \&\left(\right.$ LAT_CONFIG==1) data quality filters and applied a zenith angle cut of $90^{\circ}$ to get rid of the contamination from the Earth's atmosphere. We build the sources model database, based on the second incremental version of the fourth Fermi-LAT catalog (4FGL-DR2; Ballet et al. 7, Abdollahi et al. 1). For the Galactic diffuse emission model the (gll_iem_v07.fits) with isotropic component (iso_P8R3_ULTRACLEANVETO_V3_v1.txt) were used.

\subsection{Morphological and Spectral Studies}

Xi et al. [22] and Adam et al. [3] recently reported the detection of $\gamma$-ray emission from the direction of the Coma cluster, where they used different spatial and spectral models. We analyzed the $\gamma$-ray data for $100 \mathrm{MeV}-1 \mathrm{TeV}, 100 \mathrm{MeV}-1 \mathrm{GeV}$, and $1 \mathrm{GeV}-1 \mathrm{TeV}$ energy bands for the detailed

\footnotetext{
1https://github.com/fermi-lat/Fermitools-conda/wiki
} 

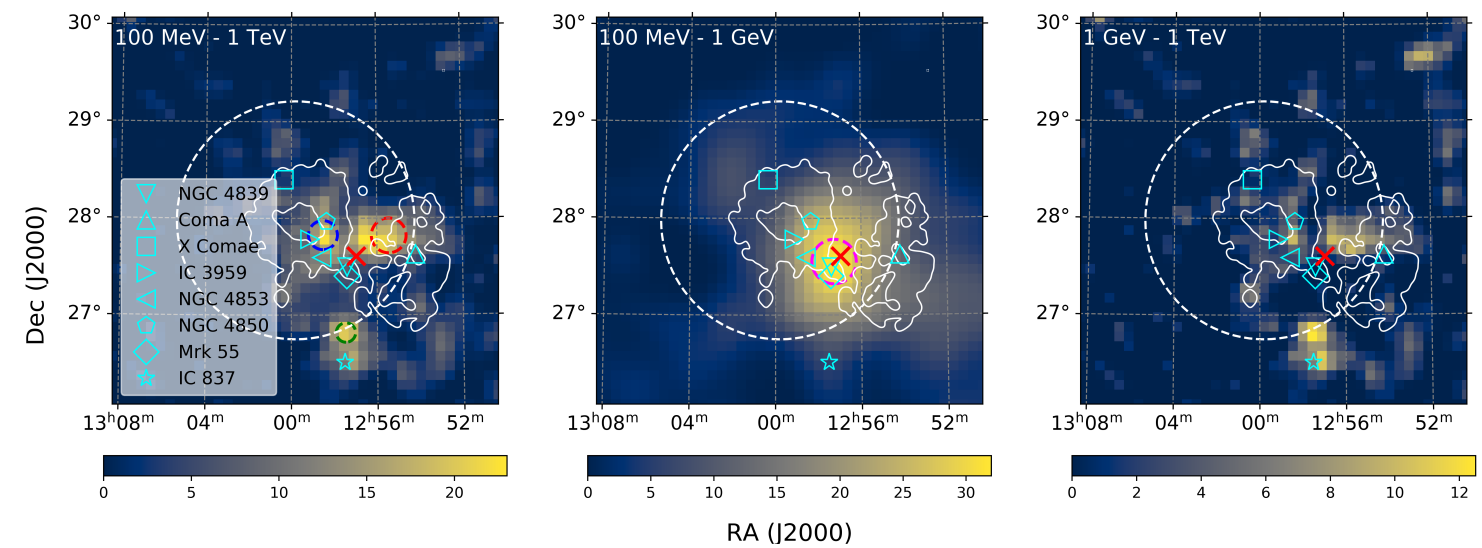

Figure 1: Significance (TS) maps of the Coma cluster region, overlaid with radio $352 \mathrm{MHz}$ contours (whitecolor; [8]. Left: $100 \mathrm{MeV}-1 \mathrm{TeV}$, middle: $100 \mathrm{MeV}-1 \mathrm{TeV}$, right: $1 \mathrm{GeV}-1 \mathrm{TeV}$ energy ranges. The dashed white circle $\left(\theta_{200}=1.23^{\circ}\right)$ is the virial region of the Coma cluster. The red marker shows the location of 4FGL J1256.9+2736 source. The blue, red, and green colored dashed circles in the left panel and magenta dashed circle in the middle panel correspond to the $95 \%$ containment radii of point-like sources around $\mathrm{p}_{1}, \mathrm{p}_{2}, \mathrm{p}_{3}$ and $\mathrm{p}_{\text {low }}$, respectively. The cyan-colored markers represent different types of AGN in the region with NASA/IPAC Extragalactic Data (NED) positions

Table 1: The best parameters for the spatial models used to model the Coma Cluster region.

\begin{tabular}{lcccccc}
\hline Spatial model & $\begin{array}{c}\mathrm{RA}_{\mathrm{J} 2000} \\
{[\mathrm{deg}]}\end{array}$ & $\begin{array}{c}\mathrm{Dec}_{\mathrm{J} 2000} \\
{[\mathrm{deg}]}\end{array}$ & $\begin{array}{c}\mathrm{R}_{68}^{\mathrm{pOS}} \\
{[\mathrm{deg}]}\end{array}$ & $\mathrm{TS}$ & $\begin{array}{c}\mathrm{R}_{68} \\
{[\mathrm{deg}]}\end{array}$ & $\mathrm{TS}_{\mathrm{ext}}$ \\
\hline RadialDisk & $194.14 \pm 0.14$ & $27.38 \pm 0.13$ & 0.19 & 56.4 & $0.82_{-0.05}^{+0.10}$ & 29.3 \\
RadialGaussian & $194.27 \pm 0.17$ & $27.56 \pm 0.17$ & 0.26 & 55.0 & $0.91_{-0.16}^{+0.18}$ & 9.8 \\
$\mathbf{p}_{\mathbf{1}}+\mathbf{p}_{\mathbf{2}}+\mathbf{p}_{\mathbf{3}}$ & & & & & & \\
$\mathrm{p}_{1}$ & $194.63 \pm 0.08$ & $27.83 \pm 0.13$ & 0.15 & 17.5 & - & - \\
$\mathrm{p}_{2}$ & $193.86 \pm 0.15$ & $27.82 \pm 0.10$ & 0.18 & 16.8 & - & - \\
$\mathrm{p}_{3}$ & $194.37 \pm 0.06$ & $26.82 \pm 0.07$ & 0.10 & 15.8 & - & - \\
\hline
\end{tabular}

morphological studies. We removed the 4FGL J1256.9+2736 from the source list and assumed different spatial models to estimate $\gamma$-ray excess from the direction of the Coma cluster. For the fitting, the spectral parameters of sources inside the ROI were left as a free parameter. After the successful fit, we used find_sources tool to find and model significant peaks with test statistic (TS) values higher than 16 . As a result, the TS maps for $100 \mathrm{MeV}-1 \mathrm{TeV}, 100 \mathrm{MeV}-1 \mathrm{GeV}$, and $1 \mathrm{GeV}-1$ $\mathrm{TeV}$ energy bands are shown in Figure 1.

The TS map $100 \mathrm{MeV}-1 \mathrm{TeV}$ (shown in the left panel of Figure 1) shows that three point-like structures are detected (hereafter: $\mathrm{p}_{1}, \mathrm{p}_{2}, \mathrm{p}_{3}$ ). The best position evaluated by find_sources tool are the following; i) $\mathrm{p}_{1}$, R.A., Dec $=(194.63,27.83)$,ii $\mathrm{p}_{2}$, R.A., Dec $=(193.86,27.82)$, and iii) $\mathrm{p}_{3}$, R.A., Dec $=(194.37,26.82)$. Interestingly, similar $\gamma$-ray structures are noticeable also for in the highest energy band $(1 \mathrm{GeV}-1 \mathrm{TeV})$, however with lower detection significance (right panel of Figure 1). The lowest energy ( $100 \mathrm{MeV}-1 \mathrm{GeV}) \mathrm{TS}$ map is depicted on the middle panel of Figure 1 , where the peak (hereafter $p_{\text {low }}$ ) in this energy band resolved with find_sources is shown by the 


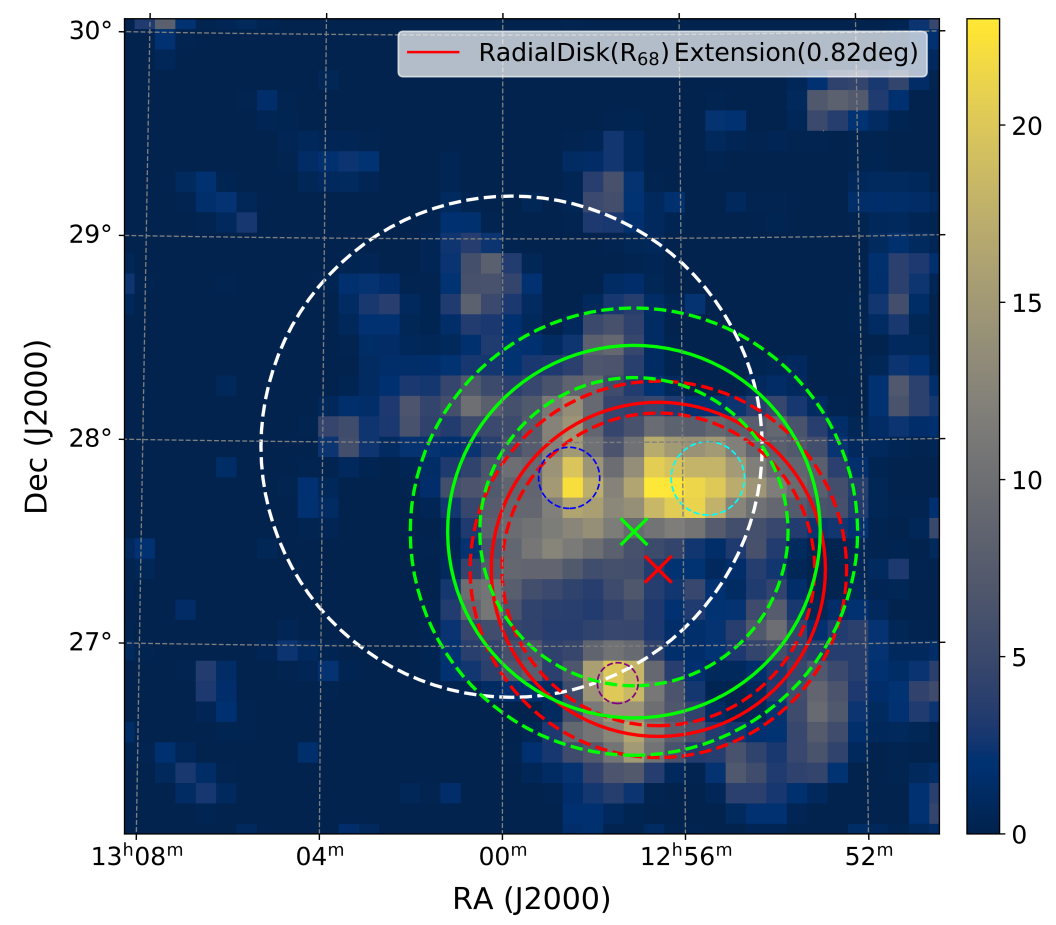

Figure 2: The TS map of the Coma Cluster for the $100 \mathrm{MeV}$ to $1 \mathrm{TeV}$ energy band. The red and green circles are the RadialDisk and RadialGaussian models, where dashed circles correspond to their errors.

dashed magenta circle, with the $68 \%$ containment radius.

It is noticeable in the TS maps in Figure 1 that the residual $\gamma$-ray map has possibly extended structure. To test of this assumption, for the whole energy range (100 MeV $-1 \mathrm{TeV})$ we performed an extension analysis on the 4FGL J1256.9+2736 source position, using extension tool in Fermipy. We assumed 2D Gaussian and 2D uniform disk spatial model templates for the extended analysis. For the $2 \mathrm{D}$ uniform disk model we obtained the following results: $\mathrm{R}_{68}=0.82_{-0.05}^{+0.10}$ containment radius with $\mathrm{TS}_{\mathrm{ext}} \sim 29.3$ for the extension significance. For the 2D Gaussian model we obtained $\mathrm{R}_{68}=0.91_{-0.16}^{+0.18}$ containment radius and $\mathrm{TS}_{\mathrm{ext}} \sim 9.8$ for the extension significance. We also tried Disk $+\mathrm{p}_{1}+\mathrm{p}_{2}+\mathrm{p}_{3}$ model to explain the the complex $\gamma$-ray excess from Coma Cluster region, which includes three point like sources and an extended diffuse component. The spatial and spectral results are shown in Table 1 and 2.

For the spectral studies we extracted the $\gamma$-ray spectrum from the extended region modeled with RadialDisk. Interestingly, the $\gamma$-ray spectrum extends up to $\sim 50 \mathrm{GeV}$ with hard photon index $\sim 2.2 \pm 0.11$. The spectrum is shown in Figure 3 .

\section{Discussion and Conclusion}

The $\gamma$-ray emission from the direction of Coma Cluster with Fermi-LAT has been studied in this paper. 12.3 years of accumulated data allowed us to investigate the $\gamma$-ray emission, by implementing detailed morphological and spectral studies. Assuming different spectral and spatial models the $\gamma$-ray excess from the direction of Coma Cluster are successfully modeled. As a result, 
Table 2: The results of the spectral parameters for each model in the $100 \mathrm{MeV}$ to $1 \mathrm{TeV}$ energy range. The Energy flux, Power-Law spectral index, detection TS, maximum-likelihood $\log (\mathcal{L})_{\max }, \Delta_{\text {AIC }}$ and $\mathrm{N}_{\text {dof }}$ values are shown in each column respectively.

\begin{tabular}{lcccccc}
\hline Spatial model & $\begin{array}{c}\text { Energy flux } \\
{\left[10^{-12} \times \mathrm{erg} \mathrm{cm}^{-2} \mathrm{~s}^{-1}\right]}\end{array}$ & Index & TS & $\log (\mathcal{L})_{\max }$ & $\Delta_{\text {AIC }}$ & $\mathrm{N}_{\text {dof }}$ \\
\hline Disk & $3.84 \pm 0.67$ & $2.23 \pm 0.11$ & 51.6 & -313141.3 & - & - \\
$\mathbf{p}_{\mathbf{1}}+\mathbf{p}_{\mathbf{2}}+\mathbf{p}_{\mathbf{3}}$ & & & & -313135.0 & -4.7 & 4 \\
$\mathrm{p}_{1}$ & $1.05 \pm 0.37$ & $2.47 \pm 0.23$ & 17.2 & & & \\
$\mathrm{p}_{2}$ & $1.16 \pm 0.39$ & $2.53 \pm 0.24$ & 16.4 & & & \\
$\mathrm{p}_{3}$ & $0.93 \pm 0.35$ & $2.08 \pm 0.24$ & 15.4 & & & \\
Disk + $\mathbf{p}_{\mathbf{1}}+\mathbf{p}_{\mathbf{2}}+\mathbf{p}_{\mathbf{3}}$ & & & & -313131.7 & -7.2 & 6 \\
Disk & $1.54 \pm 1.06$ & $2.09 \pm 0.26$ & 5.62 & & & \\
$\mathrm{p}_{1}$ & $0.82 \pm 0.37$ & $2.44 \pm 0.28$ & 11.1 & & & \\
$\mathrm{p}_{2}$ & $0.86 \pm 0.43$ & $2.56 \pm 0.32$ & 8.8 & & & \\
$\mathrm{p}_{3}$ & $0.75 \pm 0.39$ & $1.99 \pm 0.30$ & 9.4 & & & \\
\hline
\end{tabular}

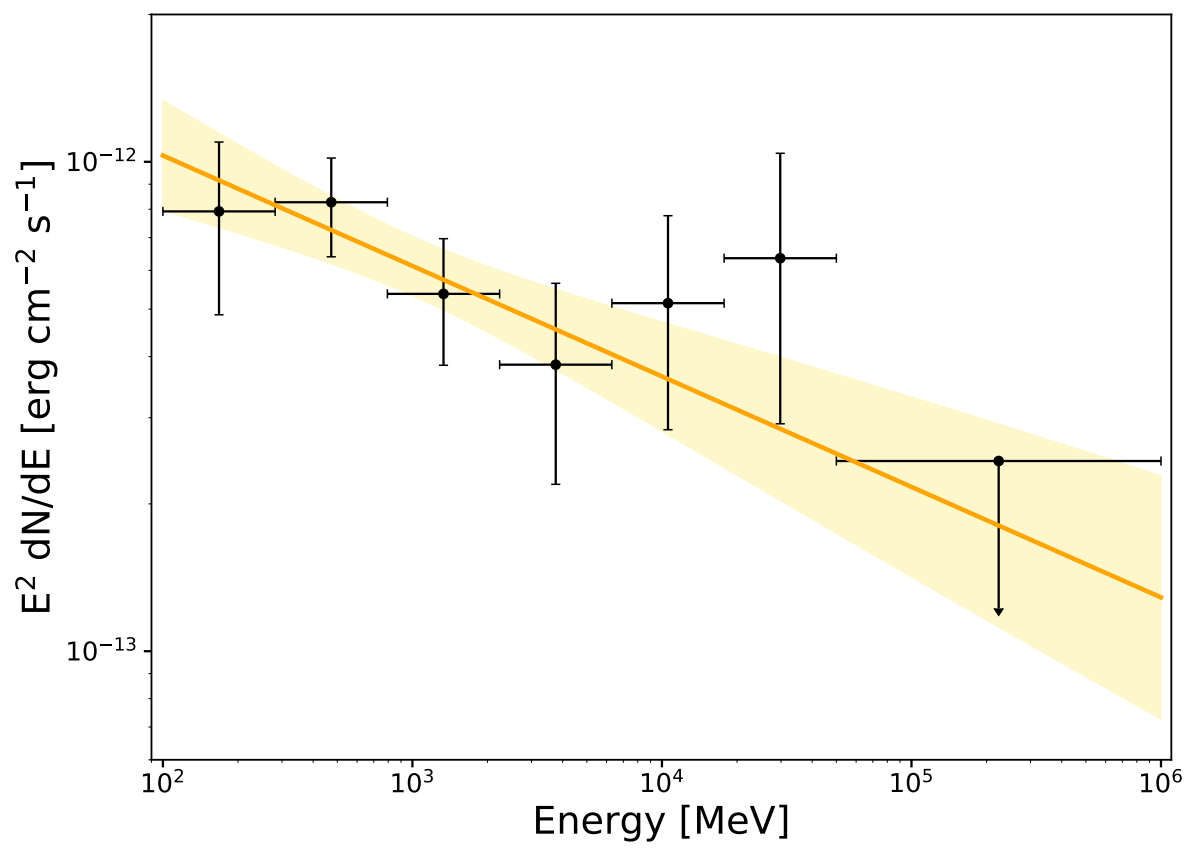

Figure 3: The SED of the $\gamma$-ray excess from the region of the Coma cluster for the RadialDisk model in black color, where the ULs are computed at $95 \%$ CL for the energy bins below $2 \sigma$. 
three point-like sources and extended diffuse $\gamma$-ray structures have been investigated. The maps are shown in Figure 1 and the spatial model fit results in Table 1. The extended $\gamma$-ray emission from the Coma region is well described by RadialDisk model with $\mathrm{R}_{68}=0.82_{-0.05}^{+0.10}$ deg extension size (with higher than $5 \sigma$ extension significance). Moreover, three point-like structures have additional improvement to explain the complex $\gamma$-ray morphology. The results are shown in Table 1 and in Table 2 and, in order to compare different models, the corresponding log-likelihood and the Akaike information criterion (AIC) values for each model are also estimated. Interestingly, the effective acceleration of the particles could produce the $\gamma$-ray spectrum that extends up to $\sim 50 \mathrm{GeV}$ with a hard photon index. The theoretical explanation of this $\gamma$-ray emission from the direction of the Coma Cluster is challenging, and different scenarios can be proposed in this regard through leptonic and hadronic radiative processes. For example, one possible scenario is that the $\gamma$-rays are initiated by inverse Compton (IC) of secondary electrons (Bethe-Heitler) pair produced at interactions of energetic protons $\left(\sim 10^{18} \mathrm{eV}\right)$ with $2.7 \mathrm{~K} \mathrm{CMBR}$ photons [13, 15].

\section{Acknowledgement}

DZ acknowledges funding from the Irish Research Council Starting Laureate Award (IRCLA/2017/83). JM acknowledges funding from funding from a Royal Society-Science Foundation Ireland University Research Fellowship (20/RS-URF-R/3712).

\section{References}

[1] Abdollahi S., et al., 2020, , 247, 33

[2] Ackermann M., et al., 2016, , 819, 149

[3] Adam R., Goksu H., Brown S., Rudnick L., Ferrari C., 2021, , 648, A60

[4] Aharonian F., et al., 2009, , 502, 437

[5] Ajello M., et al., 2009, , 690, 367

[6] Atwood W., et al., 2013, Pass 8: Toward the Full Realization of the Fermi-LAT Scientific Potential (arXiv: 1303.3514)

[7] Ballet J., Burnett T. H., Digel S. W., Lott B., 2020, arXiv e-prints, p. arXiv:2005.11208

[8] Brown S., Rudnick L., 2011, Monthly Notices of the Royal Astronomical Society, 412, 2

[9] Churazov E., Khabibullin I., Lyskova N., Sunyaev R., Bykov A. M., 2020, arXiv e-prints, p. arXiv:2012.11627

[10] Deiss B. M., Reich W., Lesch H., Wielebinski R., 1997, , 321, 55

[11] Gastaldello F., et al., 2015, , 800, 139

[12] Giovannini G., Feretti L., Venturi T., Kim K. T., Kronberg P. P., 1993, , 406, 399 
[13] Inoue S., Aharonian F. A., Sugiyama N., 2005, , 628, L9

[14] Jaffe W. J., Rudnick L., 1979, , 233, 453

[15] Kelner S. R., Aharonian F. A., 2008, , 78, 034013

[16] Large M. I., Mathewson D. S., Haslam C. G. T., 1959, , 183, 1663

[17] Lutovinov A. A., Vikhlinin A., Churazov E. M., Revnivtsev M. G., Sunyaev R. A., 2008, , 687, 968

[18] Struble M. F., Rood H. J., 1991, , 77, 363

[19] Völk H. J., Aharonian F. A., Breitschwerdt D., 1996, , 75, 279

[20] Willson M. A. G., 1970, , 151, 1

[21] Wood M., Caputo R., Charles E., Di Mauro M., Magill J., Perkins J. S., Fermi-LAT Collaboration 2017, in 35th International Cosmic Ray Conference (ICRC2017). p. 824 (arXiv:1707.09551)

[22] Xi S.-Q., Wang X.-Y., Liang Y.-F., Peng F.-K., Yang R.-Z., Liu R.-Y., 2018, , 98, 063006 